This is an electronic reprint of the original article. This reprint may differ from the original in pagination and typographic detail.

Author(s): Mtsuko, Davie; Koshio, A.; Yudasaka, M.; lijima, S.; Ahlskog, Markus

Title: $\quad$ Measurements of the transport gap in semiconducting multiwalled carbon nanotubes with varying diameter and length

Year: $\quad 2015$

Version:

Please cite the original version:

Mtsuko, D., Koshio, A., Yudasaka, M., lijima, S., \& Ahlskog, M. (2015). Measurements of the transport gap in semiconducting multiwalled carbon nanotubes with varying diameter and length. Physical Review B, 91(19), Article 195426.

https://doi.org/10.1103/PhysRevB.91.195426

All material supplied via JYX is protected by copyright and other intellectual property rights, and duplication or sale of all or part of any of the repository collections is not permitted, except that material may be duplicated by you for your research use or educational purposes in electronic or print form. You must obtain permission for any other use. Electronic or print copies may not be offered, whether for sale or otherwise to anyone who is not an authorised user. 


\title{
Measurements of the transport gap in semiconducting multiwalled carbon nanotubes with varying diameter and length
}

\author{
D. Mtsuko, ${ }^{1}$ A. Koshio, ${ }^{2}$ M. Yudasaka, ${ }^{2}$ S. Iijima, ${ }^{2}$ and M. Ahlskog ${ }^{1, *}$ \\ ${ }^{1}$ University of Jyväskylä, Nanoscience Center, P.O.B 35 (YFL), Jyväskylä, FI-40014, Finland \\ ${ }^{2}$ NEC Research Center, Tsukuba, Ibaraki 305-8501, Japan
}

(Received 26 November 2014; revised manuscript received 6 April 2015; published 20 May 2015)

\begin{abstract}
Low temperature transport in multiwalled carbon nanotubes (MWNTs) has been studied at different diameters and lengths, within $2-10 \mathrm{~nm}$, and $0.3-3.5 \mu \mathrm{m}$, respectively. In a majority of the samples, semiconductivity showed up as a transport gap in the gate voltage controlled conduction, but metallic MWNTs are found in all diameters. The transport gap is seen to be quantitatively determined by a diameter dependent band gap, and length dependent localization of charge carriers. The band gap of semiconducting MWNTs is estimated to be smaller than that extrapolated from the conventional expression applicable to semiconducting single wall carbon nanotubes. The results constitute a systematical study on size dependent transport and especially of semiconductivity in MWNTs.
\end{abstract}

DOI: 10.1103/PhysRevB.91.195426

PACS number(s): 73.63.Fg, 72.20.Ee, 72.80.Rj, 73.20.At

Multiwalled carbon nanotubes (MWNTs), which are reasonably close to the ideal structure of several concentric single walled carbon nanotubes (SWNTs), have diameters from 2 to $\sim 20 \mathrm{~nm}$. In MWNTs of larger diameters $(>20 \mathrm{~nm})$, the disorder tends to be severe, especially in the outer sections, such that the layered structure is disturbed. Therefore, the upper limit for the diameter of true MWNTs is arbitrary. At the other end of the size range, double walled carbon nanotubes (DWNTs) are the smallest and simplest form of MWNTs. Among these and especially SWNTs, both semiconducting and metallic types have been well demonstrated (depending on chirality) to have tremendous electronic and optical properties, for example, ballistic conduction [1-3]. Much progress has also been made on developing applications of SWNTs based on these properties [4].

In contrast, much less has been accomplished on MWNTs in general, at least if one restricts the view on single nanotube devices. Quite a few experimental papers have studied electronic low temperature transport [5-10] and high magnetic field properties in MWNTs [11-15]. Additionally, conductive properties have been studied in various technically elaborate experiments [16-19] that probed interlayer interactions, contact effects, etc. Presently, the prevailing understanding of transport in MWNTs sees them mainly as diffusive low dimensional metallic conductors, with very few examples of semiconducting behavior, and no consistent, experimentally verified description of semiconductivity. However, transport properties of MWNTs have to date not been probed systematically at different diameters. In particular, for reasons mainly due to synthesis technology, there have been very few reports on MWNTs with the diameter within the interesting range of 3-10 nm; the majority of the papers (excluding those on DWNTs) have dealt with tubes of diameters $\geqslant 10 \mathrm{~nm}$. This fact has left the experimental studies on MWNTs rather disconnected from those on SWNTs and DWNTs. Moreover, the present situation is confused in that, within the reported papers, the extent of disorder varies due to very different

*Corresponding author: ahlskog@jyu.fi synthesis techniques, with some demonstrating quasiballistic conduction [6] and others demonstrating transport close to strong localization [7].

In most studies on MWNTs, the working assumption has been that the outer layer (or possibly a few outer layers) is solely responsible for the low bias transport properties. One basis for this assumption is the very large anisotropy of conductance in graphite and few layer graphene. In principle, one should find among MWNT-based devices a division into metallic or semiconducting types, for example, with respect to the outer layer. In semiconducting SWNTs, for the dependence of the band gap $\left(E_{G}\right)$ on diameter $(D)$, the conventional tight-binding theory calculation gives:

$$
E_{G}=\beta / D
$$

where $\beta \approx 0.7 \mathrm{eVnm}[1,2]$. Various quantities used in this paper are explained in Table I. Thus, in a first approximation, Eq. (1) is expected to apply to the semiconducting outer layers of MWNTs. The semiconducting properties of a MWNT can be measured, at least qualitatively, in a three-terminal fieldeffect device configuration, where its band gap shows up as a transport gap, which is the range of gate voltages where the conductance decreases strongly or vanishes. Thorough reports of such measurements on semiconducting MWNTs are very few. Some can be found in connection with the studies on transport at high magnetic fields quoted above.

Very instructive in this context is the current intense research on graphene nanoribbons (GNRs), where it is sought to create a band gap via quantum confinement by narrowing the width [20-22]. To their advantage over carbon nanotubes, GNRs can be lithographically fabricated to any desired size, within technical limits. Therefore, many recent experimental papers on transport in GNRs include a significant range of different widths and lengths, down to the nanometer scale. In these papers, the size of the band gap has been found to be roughly in a similar inverse relation with the width, as in the case of the diameter dependence of semiconducting SWNTs. To their disadvantage, GNRs are both theoretically and in practice associated with disorder in the edge structure. 
TABLE I. List of the key quantities used in this paper.

\begin{tabular}{|c|c|}
\hline Quantity & Explanation \\
\hline$D$ & MWNT outer diameter \\
\hline$L$ & Separation between electrodes \\
\hline$E_{G}$ & Eq. (1) \\
\hline$V_{G}$ & Fig. 1(c) \\
\hline$R_{\mathrm{MIN}}$ & Fig. 1(c) \\
\hline$R_{\mathrm{MAX}}$ & Fig. 1(c) \\
\hline$G_{\mathrm{MAX}}$ & $1 / R_{\mathrm{MIN}}$ \\
\hline$G_{\mathrm{MIN}}$ & $1 / R_{\mathrm{MAX}}$ \\
\hline$R_{R}$ & $R_{\mathrm{MAX}} / R_{\mathrm{MIN}}$ \\
\hline$\alpha$ & Eq. (2) \& Fig. 5(a) \\
\hline$\Delta U_{B}$ & Fig. $5(\mathrm{~b})$ \\
\hline$E_{A}$ & Eq. (5) \\
\hline
\end{tabular}

Presently, a major issue that has not been explored is the occurrence of metallic and semiconducting transport types in MWNTs of different diameter. For DWNTs, a few experimental [23-27] and theoretical [1,28-31] papers have focused on their multilayer aspects, but beyond them, in both experiment and theory, numerous practical difficulties for systematic research arise. For the semiconducting MWNTs, the exact nature of the energy gap in the presence of interwall interactions, and especially as a function of the diameter, is not known. Interlayer effects can be expected to grow in relative significance as the diameter increases. A rigorously complete understanding would of course require knowing the chirality of the outer layers, but short of that, the diameter has been used as a fundamental parameter, based on Eq. (1).

In an earlier publication [10], the authors reported on diameter dependent transport in moderate diameter MWNTs, where it was found that a transport gap opens up in the gate dependent conductance for MWNTs with diameters $<10 \mathrm{~nm}$. In this paper, we extend these measurements. Three-terminal field-effect devices are fabricated from high quality MWNTs with diameters in the range from 2 to $10 \mathrm{~nm}$. We find both metallic and semiconducting behavior, but more of the latter. By including a large range of samples with different $D$ and different interelectrode separation $(L)$, we present in this paper a systematic experimental study on the presence of a transport gap in MWNT devices.

We have fabricated single MWNT field-effect devices following standard procedures where microelectrodes, acting as drain and source connections, attach to individual MWNTs, as is shown for a typical sample in Fig. 1(a). The tubes are on pieces of $\mathrm{Si} / \mathrm{SiO}_{2}$ wafer, where the highly doped $\mathrm{Si}$ acts as a backgate electrode, separated from the tube by the $\mathrm{SiO}_{2}$ layer of $300 \mathrm{~nm}$ thickness. More than $80 \mathrm{MWNTs}$ were measured. These were synthesized as reported in Ref. [32] and can be considered to be of high quality [33], comparable to or better than conventional arc-discharge synthesized MWNTs. Atomic force microscopy (AFM) imaging was performed on all measured MWNTs to ensure that the outer layer was continuous and that the measured section was clean. The fabrication and conductivity measurement methods are described in more detail in the Supplemental Material [34]. Most importantly, we categorize our devices according to their distribution in $D$ and $L$, which is shown in Fig. 1(b).
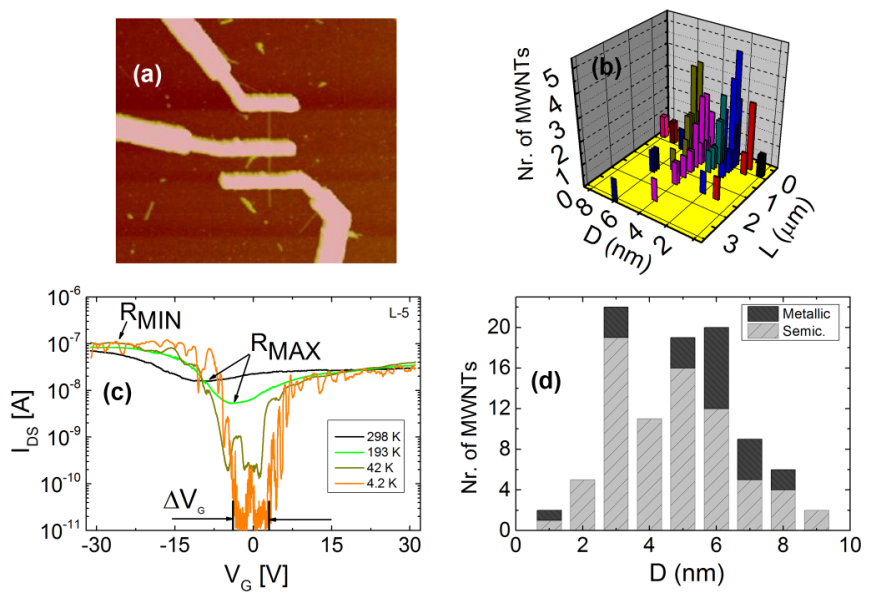

FIG. 1. (Color online) (a) AFM image of MWNT on $\mathrm{Si} / \mathrm{SiO}_{2}$, contacted by three electrodes and two different interelectrode separations $(L)$. (b) Distribution of the measured devices in this paper with respect to diameter $(D)$ and $L$. (c) Typical behavior of gate voltage $\left(V_{G}\right)$ controlled current $I_{D S}$ in a MWNT device. Included is visual definition of the on- and off-state resistances $R_{\mathrm{MIN}}$ and $R_{\mathrm{MAX}}$, and the width of the transport gap $\Delta V_{G}$. (d) Diameter distribution of semiconducting and metallic MWNT devices.

The temperature dependent gate response curves, that is drain-source current $\left(I_{D S}\right)$ at fixed bias voltage $\left(V_{D S}\right)$ vs the gate voltage $\left(V_{G}\right)$, were measured as is shown for a typical sample in Fig. 1(c), at different temperatures, typically from 300 to $4.2 \mathrm{~K}$. A gate response curve that exhibits modulation has minimum $\left(R_{\mathrm{MIN}}\right)$ and maximum $\left(R_{\mathrm{MAX}}\right)$ resistance values. The transport gap is marked in the figure and, in this case opens clearly only at low temperatures. We call these MWNTs gapped or semiconducting, with the assumption that the transport gap is due to a band gap in the electronic structure of the MWNT, as discussed below.

In Fig. 2, we show in three different devices the variety of behavior seen in the measurement data. The device in Fig. 2(a) has a transport gap in its gate response curve and is thus semiconducting. In contrast, the sample in Fig. 2(b) is metallic, with no off-state region at any temperature. In borderline cases, a very shallow off-state opens up at low temperatures, and therefore we occasionally describe some samples as quasimetallic. Figure 2(c) and 2(d) shows the temperature dependent gate response curve in a case where one MWNT is partitioned with three electrodes into two devices with different $L$ [similarly as in Fig. 1(a)]. The data clearly show the $L$ dependence of the transport gap, in that the longer $(L=0.6 \mu \mathrm{m})$ section in Fig. 2(d) exhibits a wider transport gap than the shorter $(L=0.3 \mu \mathrm{m})$ one of Fig. 2(c).

Figure 1(d) shows the $D$ distribution for MWNTs of gapped and metallic/quasimetallic character. The distribution shows that the overwhelming majority of MWNTs with $D<10 \mathrm{~nm}$ are gapped/semiconducting, which we focus on in this paper. These results allow us to report the statistics of metallicity/semiconductivity among MWNTs.

The $L$ dependence values of the on-state resistance $R_{\mathrm{MIN}}$ of a large number of samples are shown in Fig. 3, at temperatures of 300 and $4.2 \mathrm{~K}$. The $300 \mathrm{~K} R_{\text {MIN }}$ values of these nanotubes range within $13-170 \mathrm{k} \Omega$, but most are closer to the lower value. 

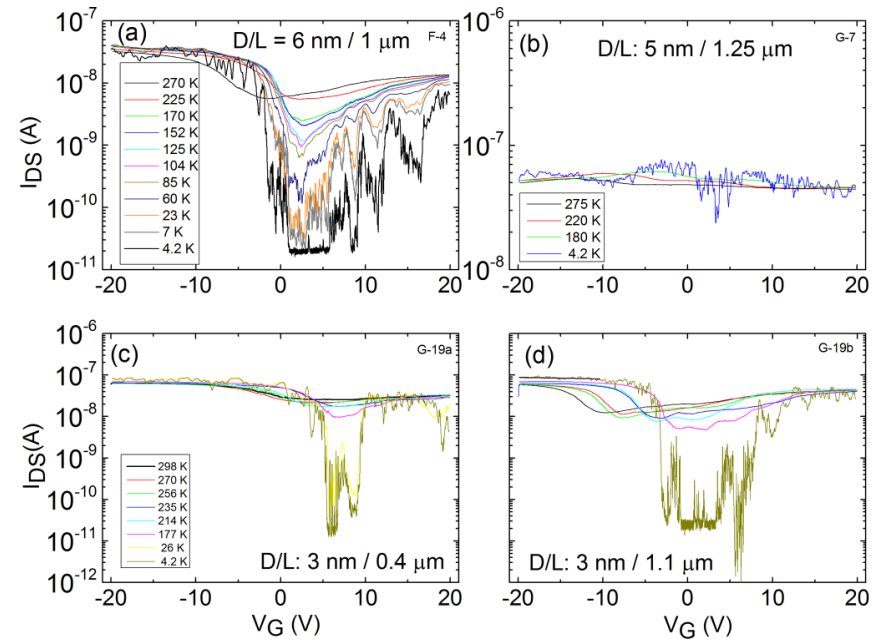

FIG. 2. (Color online) Temperature dependent gate response curves $I_{D S}$ vs $V_{G}$ (at $V_{D S}=1 \mathrm{mV}$ ) of a few different but typical cases among the measured devices of this paper. Diameter and length are indicated in each figure. The two lower graphs framed together (c) and (d) are of the same MWNT from adjacent segments with different $L$ [as in Fig. 1(a)].

The $D$ dependence of $R_{\mathrm{MIN}}$ is weak, but in general, devices with small $D$ have higher $R_{\mathrm{MIN}}$ at $300 \mathrm{~K}$ as compared to those with large $D$. On the other hand, it is observed that, as a function of $L$, the minimum $R_{\mathrm{MIN}}$ increases as the length $L$ increases beyond $1 \mu \mathrm{m}$. The dotted line in Fig. 3 indicates the lowest values for $R_{\mathrm{MIN}}$ at $300 \mathrm{~K}$ at different lengths. $R_{\mathrm{MIN}}$ is also consistently $L$ dependent in single MWNTs with multiple electrodes and different electrode separations.

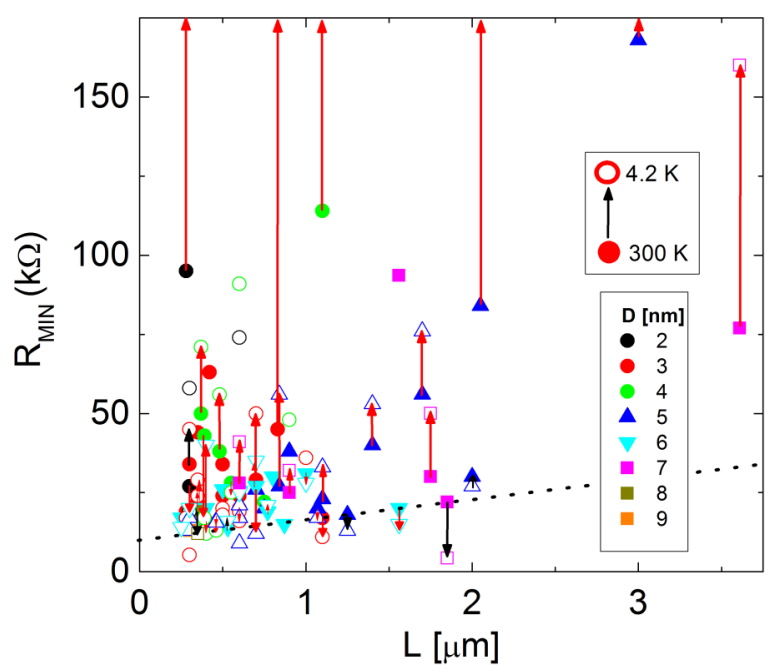

FIG. 3. (Color online) Minimum on-state resistance $R_{\text {MIN }}$ at 300 and $4.2 \mathrm{~K}$, as a function of $L$ and for different $D$, indicated in the lower inset. As the upper inset explains, the $300 \mathrm{~K}$ value is given by a filled label, the color of which indicates $D$, and which connects with an arrow to the $4.2 \mathrm{~K}$ value. Devices with a transport gap are indicated with red arrows, and metallic devices are indicated with black (metallic devices have $R_{\mathrm{MIN}} \approx R_{\mathrm{MAX}}$ ). Arrows ending at the upper line have $4.2 \mathrm{~K}$ values beyond the range of the graph. The dotted line is explained in the text.

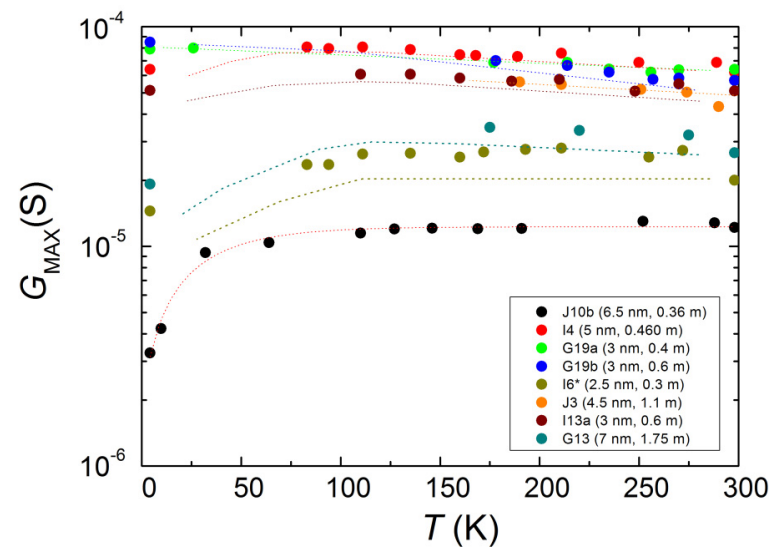

FIG. 4. (Color online) The temperature dependence of the onstate conductivity $G_{\mathrm{MAX}}=1 / R_{\mathrm{MIN}}$ for a few of the most conductive samples.

In Fig. 3, the arrows connecting the $300 \mathrm{~K}$ and the $4.2 \mathrm{~K}$ values of $R_{\text {MIN }}$ are colored according to the metallic (black) or semiconducting (red) nature as inferred from the gate response curve. At around the $300 \mathrm{~K}$ value for $R_{\mathrm{MIN}}$ of $25 \mathrm{k} \Omega$, the temperature coefficient of resistivity is about constant, and this value is a kind of demarcation line between positive values for the coefficient, for $300 \mathrm{~K} R_{\mathrm{MIN}}<25 \mathrm{k} \Omega$, and negative values for $R_{\mathrm{MIN}}>25 \mathrm{k} \Omega$. At $4.2 \mathrm{~K}, R_{\mathrm{MIN}}$ ranged for some devices to several megaohms, beyond the scale of the figure, but these are few. Figure 4 shows the temperature dependence of the conductance $G_{\mathrm{MAX}}\left(=1 / R_{\mathrm{MIN}}\right)$ of some of the most highly conducting samples.

The main topic of this paper is the transport gap in the gate response curve, as the signature of semiconductivity. We characterize the behavior of the transport gap, as a function of the $D$ - $L$ distribution given in Fig. 1(b), with the following set of parameters $\left[R_{R}, \alpha, \Delta U_{B}\right]$, which are defined as follows:

(1) The room temperature resistance ratio $R_{R}=$ $R_{\mathrm{MAX}} / R_{\mathrm{MIN}}$, where $R_{\mathrm{MAX}}$ and $R_{\mathrm{MIN}}$ are defined in Fig. 1(c).

(2) $\alpha$ is derived from the temperature dependence of $R_{\mathrm{MAX}}$, which could be obtained for samples with finite $R_{\text {MAX }}$ at room temperature, and down to such temperatures where $R_{O F F}$ was still measurable. Assuming an Arrhenius behavior $R_{\mathrm{MAX}} \sim$ $\exp ^{-\alpha / k_{B} T}$, then

$$
\log R_{\mathrm{MAX}} \sim-\alpha / k_{B} T
$$

Figure 5(a) shows the temperature dependence of $\log \left(R_{\mathrm{MAX}}\right)$ for a few samples, displayed against $1 / T$. The data follow $1 / T$ well at higher temperatures and begin to deviate from this at some lower $T$ or higher resistance; $\alpha$ is thus the slope of the line that approximates the higher end temperature behavior.

(1) The bias voltage gap $\Delta U_{B}$ is given in Fig. 5(b), which shows an $I_{D S^{-}} V_{D S}$ curve taken in the middle of the gap region. In the figure, $\Delta U_{B}$ is indicated as the width of the range of $V_{D S}$, for which $I_{D S}$ is approximately zero. We define $\Delta U_{B}$ as the maximum value reached by the bias voltage gap in the $I_{D S^{-}} V_{D S}$ characteristics as $V_{G}$ is swept across the transport gap.

A large value in the parameter set $\left[R_{R}, \alpha, \Delta U_{B}\right]$ signifies a large or "strong" gap in the transport data. Often we cannot 

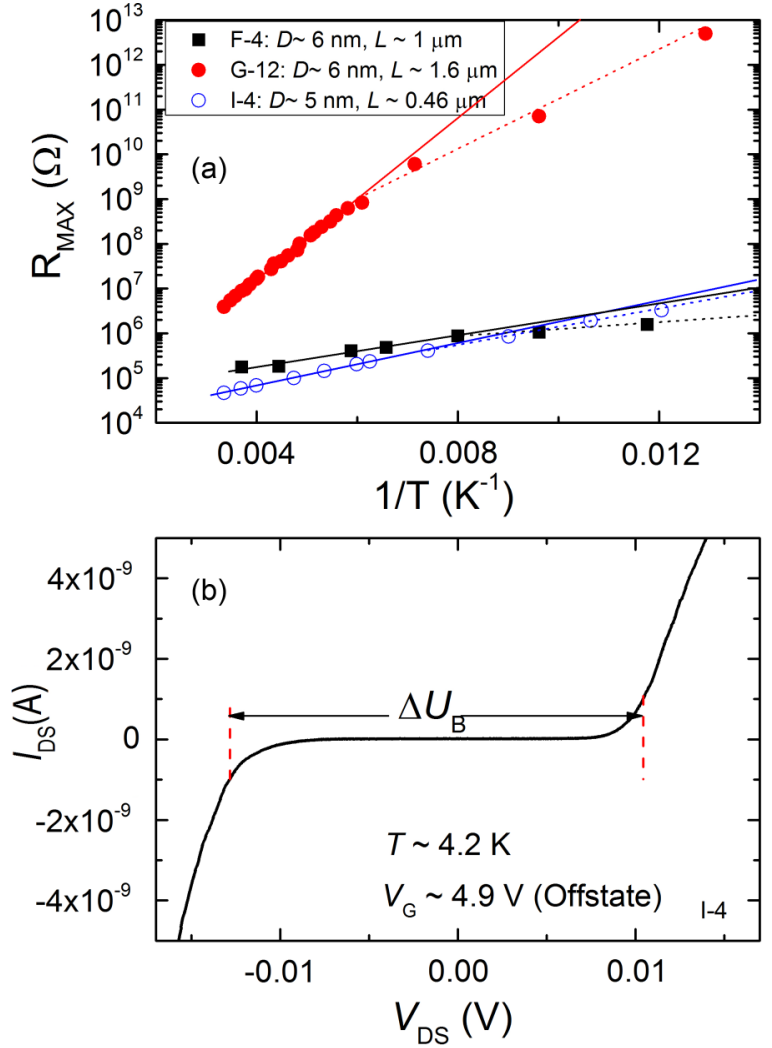

FIG. 5. (Color online) Measurement of transport gap characteristics $\alpha$ and $\Delta U_{B}$, where (a) $\alpha$ is obtained from the slope on the high temperature end of $R_{\mathrm{MAX}}$ vs $1 / T$ data [Eq. (2)]. (b) Data for three typical samples with different $D$ and $L$ are shown, where $\Delta U_{B}$ is obtained as the width of the bias voltage gap of the $I_{D S}-V_{D S}$ curve, at such value of $V_{G}$ within the transport gap that maximizes $\Delta U_{B}$.

have all three parameters for each sample. For example, the transport gap can be so strong already at room temperature that $R_{\mathrm{MAX}}(300 \mathrm{~K})$ is unmeasurable (at low bias voltage), which means that none of the following can be obtained: $R_{\mathrm{MAX}}, R_{R}$, or $\alpha$. However, in this case, $\Delta U_{B}$ should be possible to get. Conversely, if the transport gap is weak, then a clear bias voltage gap does not exist, and $\Delta U_{B}$ cannot be obtained, in which case $R_{\mathrm{MAX}}, R_{R}$, and $\alpha$ should be accessible. At a minimum, for each of our samples, we have one or more of the quantities $R_{\mathrm{MIN}}, R_{\mathrm{MAX}}, \alpha$, or $\Delta U_{B}$.

Figure 6(a) shows the data on $R_{R}$ at room temperature, and Fig. 6(b) shows the $\alpha$ values as a function of $L$ and with each diameter-defined data set shown separately. The $R_{R}$ data exhibit great variation. In the figure, for each diameter in the range 3-8 nm, the left/upper side boundary of the data points for the particular diameter is indicated with a large shaded line. A clear systematic difference between the data points of each diameter is obvious. In Fig. 6(b), the clear diameter dependence in the $\alpha$ data is also outlined with lines that separate different diameters from each other, though less obviously in this case.

The data for $\Delta U_{B}$, measured at $4.2 \mathrm{~K}$, are shown in Figs. 7(a) and 7(b), again as a function of $L$ and with each diameter defined data set shown separately. The data are this time separated into small diameter tubes [ $D \leqslant 4 \mathrm{~nm}$; Fig. 7(a)] and larger ones $[D \geqslant 5 \mathrm{~nm}$; Fig. 7(b)].
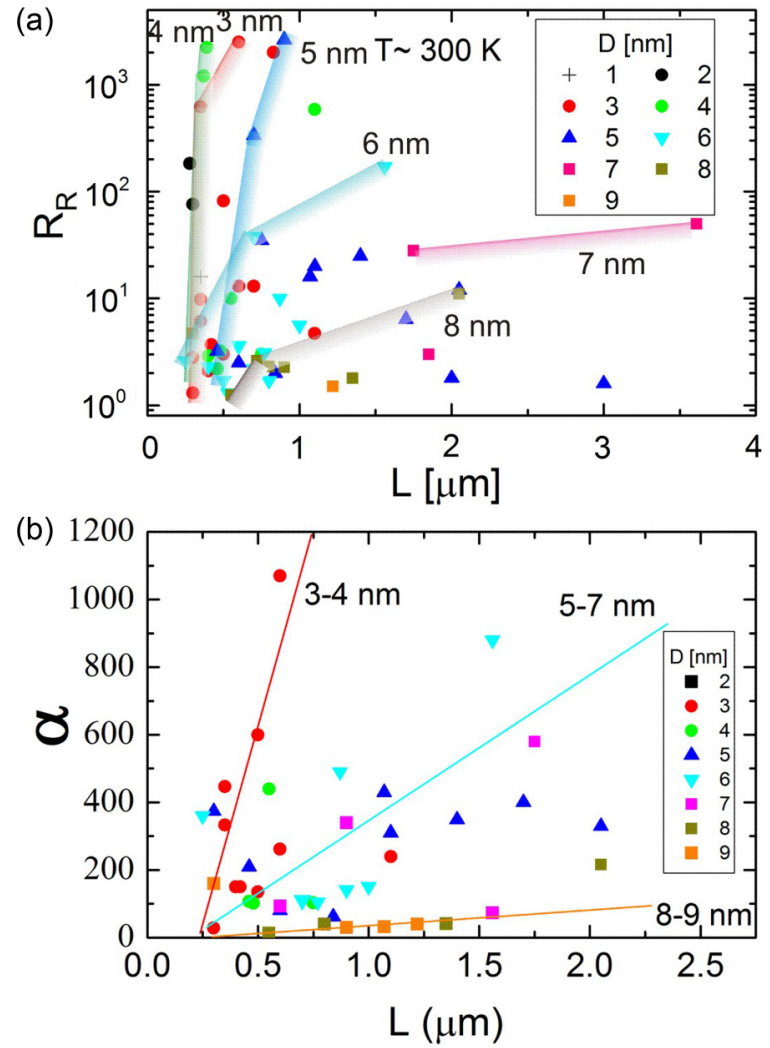

FIG. 6. (Color online) (a) Room temperature resistance ratio $R_{R}=R_{\mathrm{MAX}} / R_{\mathrm{MIN}}$ vs $L$ of MWNTs. The thick shaded lines indicate the boundary of data points for each $D$, such that points can be found only to the right/below of the line. (b) The $\alpha$ value from Eq. (2) vs $L$ for samples of different $D$, where $\alpha$ is obtained from $R_{\mathrm{MAX}}$ vs $1 / T$ measurements, displayed in Fig. 5(a).

On inspection of the data of the parameter set $\left[R_{R}, \alpha, \Delta U_{B}\right]$ in Figs. 6 and 7, we may outline the decisive trends: Among short MWNTs $(L \leqslant 0.6 \mu \mathrm{m})$, the narrow ones with $D \leqslant 4 \mathrm{~nm}$ exhibit a large variation in all three parameters, over orders of magnitude from small to large. The wider ones, with $D \geqslant 5 \mathrm{~nm}$, have only small values for the parameters. Another general feature is that especially the wider tubes $(D \geqslant 5 \mathrm{~nm})$ have a clear and substantial $L$ dependence, especially in the $\alpha$ and the $\Delta U_{B}$ data, with these quantities increasing with increasing $L$.

The general character of the $D$ dependence leads us to assume the existence of a band gap $E_{G}$ in the outer layer or layers as the fundamental entity behind the transport gap, which stands to $D$ in some inverse relation, similar to Eq. (1). In the few previous papers on semiconducting MWNTs, this relation has usually been assumed a priori. In contrast, a main goal of this paper is to compare our experimental results with this equation. Concerning the huge variation of the gap parameters $\left[R_{R}, \alpha, \Delta U_{B}\right]$ in the data on small- $D$ and short MWNTs $(D \leqslant 4 \mathrm{~nm}, L \leqslant 0.6 \mu \mathrm{m})$, we may explain the larger values by referring to the inverse- $D$ law of Eq. (1), while the small values are very possibly explained by similar curvature-induced small band gaps, which are known to exist in SWNTs of certain chiralities $[1,3]$. The $L$ dependence of the transport gap should be of similar origin in localization 

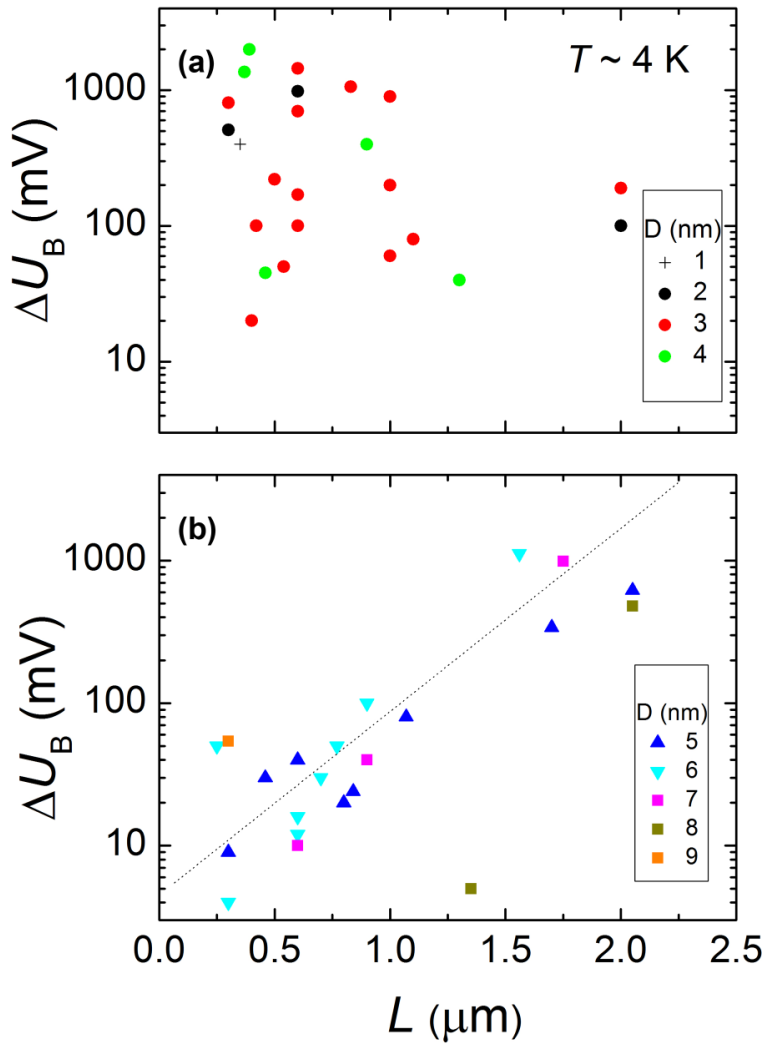

FIG. 7. (Color online) $\Delta U_{B}$ displayed for different diameters as a function of $L$, (a) for $D \leqslant 4 \mathrm{~nm}$, (b) for $D \geqslant 5 \mathrm{~nm} . \Delta U_{B}$ is defined in Fig. 5(b).

or Coulomb blockade phenomena, as has been forwarded for GNRs. We intend to analyze the size dependent data on the transport gap in order to extract an estimate for the $E_{G}$ and its diameter dependence.

The conductance $G$ may be divided into an outer layer device resistance $R_{O L}$, and an interlayer conductance $G_{I L}$, which contributes to the total conductance of a MWNT device. The temperature and gate voltage dependent $G\left(T, V_{G}\right)$ then becomes:

$$
G\left(T, V_{G}\right)=1 / R_{O L}\left(T, V_{G}\right)+G_{I L}\left(T, V_{G}\right)
$$

For the high quality MWNT structures of this paper, with an unambiguous, intact layer structure, it is clear that mobility is strongly anisotropic in favor of intralayer conduction, and that a tunneling barrier separates different layers [35-39]. The inner shell transport may be formed via thermal excitation across a gap as given by the Arrhenius equation. The tunneling probability to inner shells is in a first approximation directly proportional to the length and thus is a natural process that induces length dependence, though there are theoretical results suggesting the opposite, based on carrier momentum conservation [1]. At high biases, current diffusion to inner shells has clearly been demonstrated [40]. Thus, the approximation of exclusive outer layer transport should suffice for low bias conditions and for short devices (small $L$ ).

The data in Fig. 3 on the room temperature $R_{\text {MIN }}$ show only a slight diameter dependence, which supports the assumption of transport via the outer layer, if we further assume that in all cases, independent of diameter, the transport occurs in one-dimensional channels, which in the ballistic limit have the quantized resistance for single shell carbon nanotubes: $h / 4 e^{2}=6.5 \mathrm{k} \Omega$. In Fig. 3, the modest $L$ dependence was indicated by the dotted line, which can be fitted to the formula:

$$
R(L)=R_{C}+\rho L
$$

where $R_{C}=10.0 \mathrm{k} \Omega$, and $\rho=6.4 \mathrm{k} \Omega / \mu \mathrm{m}$. The formula expresses the idea that the resistance is composed of the contact resistance $\left(R_{C}\right)$ and a one-dimensional resistivity $(\rho)$. At $L=0$, the line extrapolates to $R_{C}$, which is only a little larger than the ballistic limit. The obtained value for $\rho$ is similar to what is typically observed in "normally defective" SWNTs [41] and also compares fairly with reported values for MWNTs. The small resistance for the majority of the samples strongly suggests a small concentration of defects and a relatively large mean free path, on the order of a few hundred nanometers, even approaching $1 \mu \mathrm{m}$. By further inspection of Fig. 3, we may observe that around the value $R_{\mathrm{MIN}} \approx 25 \mathrm{k} \Omega$ (at $300 \mathrm{~K}$ ), the sign of the temperature dependence among the samples changes. The low-temperature dependence of the conductance for some typical samples with $R_{\mathrm{MIN}}$ at or below this value was shown in Fig. 4. The positive temperature coefficient of resistance in the low-temperature limit indicates phonon scattering as the dominating mechanism, and that the mean free path is larger than the inelastic scattering length in the most conductive samples. As among the samples of Fig. 3, $R_{\mathrm{MIN}}(300 \mathrm{~K})$ becomes larger than $25 \mathrm{k} \Omega$, the temperature coefficient becomes negative, which is attributed to weak localization, and eventually the behavior turns into strong localization for the most resistive samples [1]. We note that even our gapped MWNTs exhibit generally a relatively low $R_{\mathrm{MIN}}$, and that in the shortest devices, our devices are within a quasiballistic regime.

Next, we shift to a closer analysis of the transport gap, which is the main topic of this paper. $R_{\mathrm{MAX}}\left(=1 / G_{\mathrm{MIN}}\right)$ is the resistance at the particular $V_{G}$ that sets the Fermi energy within the band gap, such that it maximizes the resistance of the MWNT. For conventional semiconductor materials, basic transport properties have of course been investigated most thoroughly [42]. In these, two parallel conduction processes appear: Band conduction via thermally activated charge carriers in extended states, and hopping conductivity via localized states, via states at the band edges that are susceptible to localization. Our situation consists of describing the transport properties of a nanoscale semiconductor, which is dimensionally constrained $(D, L)$ and which is fully susceptible to the field from the gate electrode. However, in this paper, we do not attempt to analyze the entire gate response characteristics (which contain a lot of interesting transport physics such as quantum-dot phenomena), but we limit ourselves to analyzing the behavior of $R_{\mathrm{MAX}}$, which is done in order to estimate the band gap and its size dependence. The thermally activated band conduction is described by the Arrhenius term $\exp ^{-E_{A} / k_{B} T}$, where $E_{A}$ is the activation energy. Hopping conductivity has the general expression: $\exp ^{\left(-T_{0} / T\right)^{\mu}}$, where $\mu(0<\mu<1)$ is an exponent dependent on the particular hopping mechanism, and $T_{0}$ is a factor dependent on the density of states and the localization length [43]. Applying the two factors to the case of a semiconducting MWNT outer layer, the combined 
conductance expression for $G_{\mathrm{MIN}}$ then becomes:

$$
G_{\mathrm{MIN}}=1 / R_{O L}=a \exp ^{-E_{A} / k_{B} T}+b \exp ^{\left(-T_{0} / T\right)^{\mu}}
$$

Here, $a$ and $b$ are prefactors. Generally, the band conduction term does not necessarily have a length dependence, if the object is of mesoscopic scale, which is set by the dominating scattering length. On the other hand, the hopping conduction mechanism contains an inherently length dependent resistance (for samples thinner than the hopping length, direct tunneling would occur). In typical semiconductors, the Arrhenius behavior due to thermal excitation over the band gap dominates at higher temperatures, while at lower temperatures, the hopping conduction becomes dominant. The transition temperature depends on defect/dopant density and other factors. In the data of $G_{\text {MIN }}$ for the different devices, Fig. 5(a), with different $L$, and in all measured cases, there is a deviation from the Arrhenius behavior as the temperature is decreased. This deviation is within the model of Eq. (5) accounted for by the hopping term. The deviation becomes apparent at higher temperatures (the higher the temperature, the larger the $L$ ), which thus points towards the increasing importance of the hopping contribution with increasing length.

The situation where a gate electrode controls the current through a transport gap in a single nanotube, mainly SWNTs, has been studied in many experimental papers. In some of these that consider the barrier size of the gap [44,45], $G_{\mathrm{MIN}}$ is seen to be determined by thermal activation across the band gap of the outer layer. In other words, the first expression in Eq. (5) will account alone for the conduction, giving $G_{\mathrm{MIN}}=$ $a \exp ^{-E_{A} / k_{B} T}$, which is the same as Eq. (2), which we used to extract the $\alpha$ parameter. Based on our previous discussion, this simplification can be justified, if the device length $L$ is small enough, so that quasiballistic conduction prevails.

In the multitude of low temperature experiments that have been performed on nanotube devices that exhibit ballistic behavior, including those with clean quantum-dot behavior, the interelectrode separations have typically been below $\sim 0.6$ $\mu \mathrm{m}$ [46]. Our data sets cover MWNTs in the $L$ range of $0.3-3.5 \mu \mathrm{m}$. As discussed above on our MWNT devices, the conductance data from Fig. 3 are consistent with scattering lengths of around a few hundred nanometers, which suggest the quasiballistic nature of transport in the short $(L \leqslant 0.6 \mu \mathrm{m})$ MWNT devices of this paper. Other evidence can be found in Ref. [10], where we presented Coulomb blockade oscillations at the "edges" of the transport gap in MWNTs. As we show in the Supplemental Material [34], the oscillations are typically regular for device lengths up to $0.6 \mu \mathrm{m}$ (many of our devices have this $L$ value), but irregular for devices with larger $L$, which further supports the conclusion above.

We thus assume that for short devices $(L<0.6 \mu \mathrm{m})$, with solely the Arrhenius term in Eq. (5) for $G_{\mathrm{MIN}}=1 / R_{O L}$, the $\alpha$ values obtained from Fig. 6(b) will then translate directly into values for $E_{A}=E_{G}$. We can also assume that for these, $\Delta U_{B}$ roughly corresponds to $E_{G}$, as has been assumed in Ref. [47] for GNR devices. The $\alpha$ and $\Delta U_{B}$ values (in energy units) for short MWNT devices are displayed in Fig. 8, as a function of $D$. For comparison, the figure indicates the theoretical single shell value for $E_{G}$ of Eq. (1). There is large scatter in the results, for which two primary reasons are evident: First, some of the $\Delta U_{B}$ values deviate because there is still length dependence

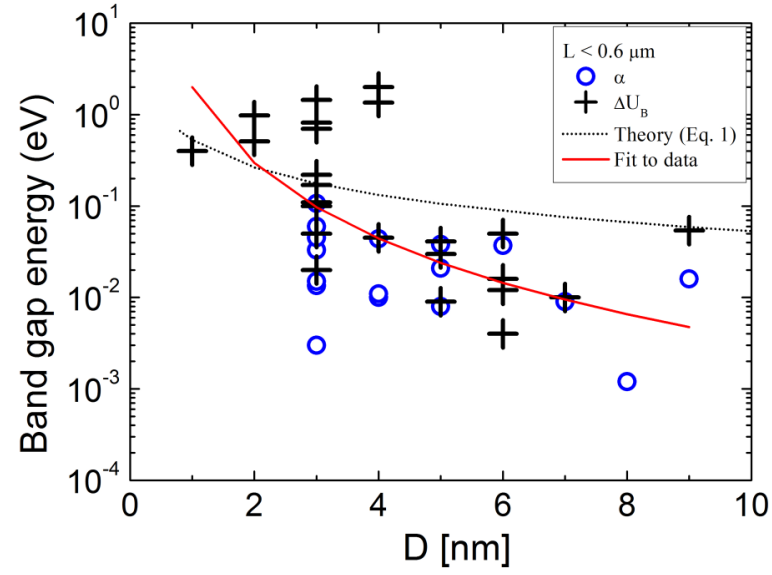

FIG. 8. (Color online) The band gap energy estimated from experimental values of $\alpha$ and $\Delta U_{B}$ vs diameter $D$, for the short $(L \leqslant 0.6 \mu \mathrm{m})$ tubes. The red line is a fit to the data. The black dotted line shows the standard tight-binding theory value for the diameter dependent band gap $E_{G}$ of semiconducting SWNTs [Eq. (1)]

in the data set (the longer samples, $L \approx 0.6 \mu \mathrm{m}$, deviate the most). Second, especially concerning the narrow $(D \leqslant 4 \mathrm{~nm})$ samples, they likely contain such small band gaps, which we briefly discussed above, that disperse the data. Nevertheless, the $\alpha$ and $\Delta U_{B}$ values roughly agree and follow a similar consistent discernible dependence on $D$. Overall, the results strongly imply that the band gap value $E_{G}$ possessed by the outer layer or layers of the MWNT devices is clearly smaller than that of the single shell value of Eq. (1), when the diameter grows well beyond those of SWNTs and DWNTs. Due to the large scatter in the data, we do not attempt to fit any functional form to it. Extrapolating the data to $D \geqslant 10 \mathrm{~nm}$, it is seen that $E_{G}$ becomes small and susceptible to thermally induced smearing of the electronic band structure, and thus the difference between metallic and semiconducting MWNTs is not observable at the usual experimentally observable temperatures or is overridden by effects stemming from the omnipresent structural disorder. This explains the situation with previous papers on transport measurements in MWNTs that we mentioned in the introduction, namely, that there were no consistent results on semiconductivity in these; the effects of the band gap in high quality MWNTs are poorly discernible if the diameter is above $10 \mathrm{~nm}$, as is the case with nearly all previous papers.

In the whole range of $L$-values, there is $L$ dependence of $\alpha$, $\Delta U_{B}$, and of the width of the transport gap, which all increase with $L$. For the longer samples, the interpretation of $\alpha$ and $\Delta U_{B}$ becomes more complicated, as both $D$ - and $L$-related effects are directly involved. As the length increases, the second term in Eq. (5), the hopping term, becomes increasingly dominant for the transport within the transport gap. There are different theoretical models for hopping conduction, some of which have $\mu=1$, and thus they are not easily separated from the Arrhenius behavior. However, generally, in this view, the nonlinearly driven hopping conduction current at high fields contributes to the bias voltage gap $\Delta U_{B}$. Moreover, inner shell conduction is certainly involved at higher voltages and as the length increases. A concise method to analyze the bias voltage gap in longer GNR devices was given in Ref. [48], where the electric field $F$ that corresponds to the $\Delta U_{B}$ was related with 
the so-called effective temperature [49] $T_{\text {eff }}$ of the hopping conduction:

$$
k_{B} T_{\text {eff }}=e F L_{c}=e\left(\Delta U_{B} / 2\right) L_{c} / L
$$

Here, $L_{C}$ is the average hopping length between localized states. To apply the same procedure to our devices, we take as $T_{\text {eff }}$ the observed deviation from the Arrhenius type $1 / T$ law. To obtain an estimate for $L_{C}$, we insert numbers for typical samples that have a clear bias voltage gap, where $L \geqslant$ $1 \mu \mathrm{m}$, and $D \leqslant 7 \mathrm{~nm}$ (Fig. 7). With $T_{\text {eff }} \sim 100 \mathrm{~K}, L \approx 1 \mu \mathrm{m}$, $\Delta U_{B} \sim 1 \mathrm{~V}$, we obtain for typical samples $L_{C} \sim 10 \mathrm{~nm}$. We can conclude that this estimate supports the argument that at lengths $\geqslant 1 \mu \mathrm{m}$, the residual conduction within the transport gap involves strong localization phenomena. We note here that the alternative model of serial Coulomb blockade, which has been utilized to explain the transport gap in GNRs, also has been forwarded to describe strongly resistive low temperature transport in SWNTs [50].

We have thus shown that MWNTs at all diameters are divided with respect to their transport properties into semiconducting and metallic types, with the former being much more prevalent. The transport gap is fundamentally due to a diameter dependent band gap, which is well observable up to $D=10 \mathrm{~nm}$, but smeared out for larger MWNTs by thermal and disorder effects. Our results suggest that quantitatively, the MWNT band gap is substantially smaller than what is given by the tight-binding theory form given in Eq. (1) for SWNTs. We will conclude this paper by briefly discussing some specific but significant research issues involving MWNTs, in light of the results of this paper.

To our knowledge, there is presently no theoretical description explicitly for the outer-layer energy gap of MWNTs of varying diameter. As we mentioned in the introduction, a few theoretical papers have addressed the issue of interlayer interaction in DWNTs, but the quantitative ramifications of this interaction will certainly change as the diameter of the MWNT grows, and conceivably more than the two outermost layers may affect even the low bias transport properties. The main result from our paper is the experimental evidence for an outer layer energy gap, which we estimated to differ significantly from Eq. (1). To this difference, a conceivable explanation could be found from the said interlayer interactions.

The band gap of semiconducting MWNTs can be modulated by high magnetic fields, as has been demonstrated in a few experimental papers $[14,15]$. While the effect has been clearly demonstrated qualitatively, the quantitative analysis rests on certain assumed parameters. In particular, in some of those papers, the same Eq. (1) of this paper, applied to the outer layer of the measured MWNT, is assumed a priori to constitute the semiconducting $E_{G}$ value of the MWNT. Moreover, some of these experiments have been carried out with very large $L$ values, which, as we have shown here, substantially entangle the effects stemming from the energy gap and localization phenomena. Therefore, the technique of these papers does not as yet provide a complete probe of the $E_{G}$ in MWNTs.

A third issue is that of MWNT quality. Our judgment, that the MWNTs used in this paper are of high quality, is based on the very uniform thickness and absolute straightness apparent from the AFM images, and this is further supported by Refs. [32,33]. On the other hand, chemical vapor deposition
CVD-grown MWNTs have an intrinsically curved shape compared to the MWNTs of this paper and arc-discharge grown MWNTs in general (Supplemental Material, Fig. S3 [34]). The curvature is seen to be more or less directly proportional to the defectiveness, and arc-discharge MWNTs are believed to be significantly less defective/disordered than the CVD-grown MWNTs. Transport in CVD-grown tubes of diameter $>10 \mathrm{~nm}$ has been studied in Ref. [7], where these were found to be close to strong localization. There are strongly different mechanical properties in arc-discharge and CVD MWNTs according to Ref. [51]. Thus there is significant variation in the properties of MWNTs, and this paper represents the domain of well ordered, relatively close to ideal MWNT structures. On the other hand, CVD-grown tubes are presently the only viable option in areas that aim for applications and mass production of MWNTs. The precise structural difference between the different classes of MWNTs has not been given much attention, although it is certainly of significance.

Finally, this paper contains a set of data of size dependent transport behavior in carbon nanotubes, which enables a comparison to papers on size dependent behavior in GNRs. GNRs always exhibit a transport gap as the width of them is reduced down to the range of a few or a few tens of nanometers $[48,52,53]$, possibly with the exception for some very recent papers [22]. This is in contrast to the MWNTs of this paper, which also include metallic transport at all diameters, and of course, it has been known since the early days of carbon nanotube research that SWNTs can be both metallic and semiconducting conductors, although, according to Ref. [3], the truly metallic ones are a very small share. Besides the difference in topology, the presence of edges and edge disorder in GNRs can be seen as the primary factor distinguishing their transport characteristics from that of carbon nanotubes. The transport gap in GNRs is seen to be caused by quantum confinement or alternatively by serial Coulomb blockade or Anderson localization, but there is presently not an effective consensus on this issue.

In summary, we have undertaken a systematic experimental investigation of the basic transport properties of MWNTs of different diameters in the range $2-10 \mathrm{~nm}$, and also of different lengths. The study has above all highlighted the size dependent transport gap, which hitherto has not been thoroughly addressed in either theoretical or experimental papers on MWNTs. Semiconducting transport behavior is more prevalent, but metallic MWNTs are also found in all diameters. An estimate was obtained for the diameter dependence of the band gap in the semiconducting MWNTs, which shows it to be substantially smaller than the conventional tight-binding theory value for single shell carbon nanotubes. The paper will hopefully help theoretical investigations on interlayer interactions in the electronic properties of MWNTs, and may also have implications to current work on GNRs.

\section{ACKNOWLEDGMENTS}

This paper was supported by the Academy of Finland (Grant No. SA-7122008). We thank Claudia Gomes da Rocha, Trinity College Dublin, for constructive discussions. 
[1] J.-C. Charlier, X. Blase, and S. Roche, Rev. Mod. Phys. 79, 677 (2007).

[2] A. Jorio, M. Dresselhaus, and G. Dresselhaus, Eds., Carbon Nanotubes (Springer, Berlin, 2008).

[3] E. A. Laid, F. Kuemmeth, G. Steele, K. Grove-Rasmussen, J. Nygård, K. Flensberg, and L. P. Kouwenhoven, arXiv:1403.6113 (to be published).

[4] Y. Che, H. Chen, H. Gui, J. Liu, B. Liu, and C. Zhou, Semicond. Sci. Technol. 29, 073001 (2014).

[5] C. Schönenberger, A. Bachtold, C. Strunk, J.-P. Salvetat, and L. Forró, Appl. Phys. A 69, 283 (1999).

[6] M. R. Buitelaar, A. Bachtold, T. Nussbaumer, M. Iqbal, and C. Schönenberger, Phys. Rev. Lett. 88, 156801 (2002).

[7] R. Tarkiainen, M. Ahlskog, A. Zyuzin, P. Hakonen, and M. Paalanen, Phys. Rev. B 69, 033402 (2004).

[8] A. Kanda, K. Tsukagoshi, Y. Aoyagi, and Y. Ootuka, Phys. Rev. Lett. 92, 036801 (2004).

[9] B. Stojetz, C. Miko, L. Forró, and C. Strunk, Phys. Rev. Lett. 94, 186802 (2005).

[10] M. Ahlskog, O. Herranen, A. Johansson, J. Leppäniemi, and D. Mtsuko, Phys. Rev. B 79, 155408 (2009).

[11] B. Lassagne, J.-P. Cleuziou, S. Nanot, W. Escoffier, R. Avriller, S. Roche, L. Forró, B. Raquet, and J.-M. Broto, Phys. Rev. Lett. 98, 176802 (2007).

[12] B. Raquet, R. Avriller, B. Lassagne, S. Nanot, W. Escoffier, J.-M. Broto, and S. Roche, Phys. Rev. Lett. 101, 046803 (2008).

[13] S. Nanot, R. Avriller, W. Escoffier, J.-M. Broto, S. Roche, and B. Raquet, Phys. Rev. Lett. 103, 256801 (2009).

[14] G. Federov, P. Barbara, D. Smirnov, D. Jimenez, and S. Roche, Appl. Phys. Lett. 96, 132101 (2010).

[15] S. H. Jhang, M. Margańska, Y. Skourski, D. Preusche, M. Grifoni, J. Wosnitza, and C. Strunk, Phys. Rev. Lett. 106, 096802 (2011).

[16] S. Frank, P. Poncharal, Z. L. Wang, and W. A. de Heer, Science 280, 1744 (1998).

[17] B. Bourlon, C. Miko, L. Forró, D. C. Glattli, and A. Bachtold, Phys. Rev. Lett. 93, 176806 (2004).

[18] J. Cumings and A. Zettl, Phys. Rev. Lett. 93, 086801 (2004).

[19] J. Y. Huang, S. Chen, S. H. Jo, Z. Wang, D. X. Han, G. Chen, M. S. Dresselhaus, and Z. F. Ren, Phys. Rev. Lett. 94, 236802 (2005).

[20] X. Wang, Y. Ouyang, L. Jiao, H. Wang, L. Xie, J. Wu, J. Guo, and H. Dai, Nature Nanotechnology 6, 563 (2011).

[21] A. Narita, X. L. Feng, Y. Hernandez, S. A. Jensen, M. Bonn, H. F. Yang, I. A. Verzhbitskiy, C. Casiraghi, M. R. Hansen, A. H. R. Koch, G. Fytas, O. Ivasenko, B. Li, K. S. Mali, T. Balandina, S. Mahesh, S. De Feyter, and K. Müllen, Nature Chemistry 6, 126 (2014).

[22] J. Baringhaus, M. Ruan, F. Edler, A. Tejeda, M. Sicot, A. Taleb-Ibrahimi, A.-P. Li, Z. Jiang, E. H. Conrad, C. Berger, C. Tegenkamp, and W. A. de Heer, Nature 506, 349 (2014).

[23] S. Moon, W. Song, J. S. Lee, N. Kim, J. Kim, S.-G. Lee, and M.-S. Choi, Phys. Rev. Lett. 99, 176804 (2007).

[24] F. Villalpando-Paez, H. Son, D. Nezich, Y. P. Hsieh, J. Kong, Y. A. Kim, D. Shimamoto, H. Muramatsu, T. Hayashi, M. Endo, M. Terrones, and M. S. Dresselhaus, Nano Lett. 8, 3879 (2008).

[25] Y. Tison, C. E. Giusca, J. Sloan, S. Ravi, and P. Silva, ACS Nano 2, 2113 (2008).
[26] K. Schouteden, A. Volodin, D. A. Muzychenko, M. P. Chowdhury, A. Fonseca, J. B. Nagy, and C. Van Hasendonck, Nanotechnology 21, 485401 (2010).

[27] D. Bouilly, J. Cabana, F. Meunier, M. Desjardins-Carriere, F. Lapointe, P. Gagnon, F. L. Larouche, E. Adam, M. Paillet, and R. Martel, ACS Nano 5, 4927 (2011).

[28] Y.-K. Kwon and D. Tomanek, Phys. Rev. B 58, R16001(R) (1998).

[29] A. Hansson and S. Stafström, Phys. Rev. B 67, 075406 (2003).

[30] D. V. Makaev and P. N. D'Yachkov, JETP Lett. 84, 335 (2006).

[31] V. Zolyomi, J. Koltai, A. Rusznyak, J. Kurti, A. Gali, F. Simon, H. Kuzmany, A. Szabados, and P. R. Surjan, Phys. Rev. B. 77, 245403 (2008).

[32] A. Koshio, M. Yudasaka, and S. Iijima, Chem. Phys. Lett. 356, 595 (2002).

[33] A. Koshio, M. Yudasaka, and S. Iijima, J. Phys. Chem. C 111, 10 (2007).

[34] See Supplemental Material at http://link.aps.org/supplemental/ 10.1103/PhysRevB.91.195426 for detailed MWNT fabrication and conductivity measurement methods.

[35] S. Roche, F. Triozon, A. Rubio, and D. Mayou, Phys. Rev. B 64, 121401 (2001).

[36] Y.-G. Yoon, P. Delaney, and S. G. Louie, Phys. Rev. B 66, 073407 (2002).

[37] F. Triozon, S. Roche, A. Rubio, and D. Mayou, Phys. Rev. B. 69, 121410 (2004).

[38] M. A. Tunney and N. R. Cooper, Phys. Rev. B 74, 075406 (2006).

[39] S. Wang, M. Grifoni, and S. Roche, Phys. Rev. B 74, 121407 (2006).

[40] P. G. Collins, M. Hersam, M. Arnold, R. Martel, and P. Avouris, Phys. Rev. Lett. 86, 3128 (2001).

[41] M. S. Purewal, B. H. Hong, A. Ravi, B. Chandra, J. Hone, and P. Kim, Phys. Rev. Lett. 98, 186808 (2007).

[42] B. I. Shklovskii and A. I. Efros, Electronic Properties of Doped Semiconductors (Springer-Verlag, 1984).

[43] A. S. Rodin and M. M. Fogler, Phys. Rev. B 84, 125447 (2011).

[44] E. D. Minot, Y. Yaish, V. Sazonova, and P. L. McEuen, Nature 428, 536 (2004).

[45] C. Zhou, J. Kong, and H. Dai, Phys. Rev. Lett. 84, 5604 (2000).

[46] F. Kuemmeth, S. Ilani, D. C. Ralph, and P. L. McEuen, Nature 452, 448 (2008).

[47] M. Y. Han, B. Özyilmaz, Y. Zhang, and P. Kim, Phys. Rev. Lett. 98, 206805 (2007).

[48] M. Y. Han, J. C. Brant, and P. Kim, Phys. Rev. Lett. 104, 056801 (2010).

[49] S. Marianer and B. I. Shklovskii, Phys. Rev. B 46, 13100 (1992).

[50] B. Gao, D. C. Glattli, B. Placais, and A. Bachtold, Phys. Rev. B 74, 085410 (2006).

[51] H. Jackman, P. Krakhmalev, and K. Svensson, Appl. Phys. Lett. 104, 021910 (2014).

[52] P. Gallagher, K. Todd, and D. Goldhaber-Gordon, Phys. Rev. B 81, 115409 (2010).

[53] J. Güttinger, F. Molitor, C. Stampfer, S. Schnez, A. Jacobsen, S. Dröscher, T. Ihn, and K. Ensslin, Rep. Prog. Phys. 75, 126502 (2012). 